\title{
NOD2 deletion promotes cardiac hypertrophy and fibrosis induced by pressure overload
}

Jing Zong ${ }^{1,2}$, Mohamed Salim ${ }^{1,2}$, Heng Zhou ${ }^{1,2}$, Zhou-yan Bian ${ }^{1,2}$, Jia Dai ${ }^{1,2}$, Yuan Yuan ${ }^{1,2}$, Wei Deng ${ }^{1,2}$, Jie-yu Zhang ${ }^{1,2}$, Rui Zhang ${ }^{1,2}$, Qing-qing $\mathrm{Wu}^{1,2}$ and Qi-zhu Tang ${ }^{1,2}$

Nucleotide-binding oligomerization domain-2 (NOD2, also designated CARD15), a member of the NOD-leucine-rich repeat (LRR) protein family (also called the CATERPILLAR family), is upregulated in atheroma lesions and has an important role in regulating the intracellular recognition of bacterial components by immune cells. However, the effect of NOD2 on cardiac hypertrophy induced by a pathological stimulus has not been determined. Here, we investigated the effects of NOD2 deficiency on cardiac hypertrophy induced by aortic banding (AB) in mice. Cardiac hypertrophy was evaluated by echocardiographic, hemodynamic, pathological, and molecular analyses. NOD2 expression was upregulated in cardiomyocytes after aortic banding surgery in wild-type (WT) mice. NOD2 deficiency promoted cardiac hypertrophy and fibrosis 4 weeks after AB. Further, the enhanced activation of TLR4 and the MAPKs, NF- $\kappa$ B and TGF- $\beta /$ Smad pathways were found in NOD2-knockout (KO) mice compared with WT mice. Our results suggest that NOD2 attenuates cardiac hypertrophy and fibrosis via regulation of multiple pathways.

Laboratory Investigation (2013) 93, 1128-1136; doi:10.1038/labinvest.2013.99; published online 19 August 2013

KEYWORDS: cardiac hypertrophy; fibrosis; MAPK; NOD2; TLR4; Smad; NF- $\kappa$ B

Cardiac hypertrophy occurs as an adaptive response to various cardiovascular diseases, such as ischemic heart disease, hypertension, valvular insufficiency, infectious agents, or mutations in sarcomeric genes. ${ }^{1}$ Although pathologic hypertrophic hearts temporarily maintain output, sustained cardiac hypertrophy can predispose a patient to arrhythmia and sudden death, and also cause heart failure. ${ }^{2,3}$ The transition between pathological cardiac hypertrophy and heart failure is associated with a reduction in cardiac contractility, ventricular remodeling, and cardiac fibrosis, as well as with myocyte loss. ${ }^{4}$ Increasing evidence shows that a number of signaling pathways have a critical role in the development of cardiac hypertrophy. These signaling pathways include the mitogen-activated protein kinase (MAPK), ${ }^{5}$ phosphoinositide 3-kinase (PI3K)/Akt, ${ }^{6} \mathrm{NF}-\kappa \mathrm{B},{ }^{7}$ and TGF- $\beta 1 /$ Smad pathways. ${ }^{8}$ However, the mechanisms that regulate these pathways have not been clearly illustrated. Therefore, it is important to elucidate the underlying mechanism of cardiac hypertrophy.

Nucleotide-binding oligomerization domain-2 (NOD2, also designated CARD15) is a member of the NODleucine-rich repeat (LRR) protein family (also called the
CATERPILLAR family). The members of the LRR protein family share a tripartite domain structure consisting of a C-terminal domain containing multiple leucine-rich repeats (LRRs), a central nucleotide-binding oligomerization domain (NOD), and an N-terminal protein-protein interaction domain, such as caspase recruitment domains (CARDs), death effector domain (DED), and pyrin domain (PYD). ${ }^{9-11}$ NOD2 has been shown to recognize muramyl dipeptide (MDP), an intracellular sensor of bacterial cell wall peptidoglycan (PGN). ${ }^{12,13}$ This activation by PGN components initiates various signaling pathways, including NF- $\kappa \mathrm{B}$ and MAPKs pathways, ultimately leading to a variety of immune responses. ${ }^{14}$ Growing evidence suggests that NOD2 mutations increase susceptibility to Crohn's disease $(\mathrm{CD})^{15}$ and are associated with Blau syndrome. ${ }^{16}$ NOD2 is expressed mainly in myeloid cells and is important in the immune response to pathogenic organisms including Mycobacterium tuberculosis and Toxoplasmosis gondii. ${ }^{17}$ However, the effect of NOD2 deficiency on cardiac hypertrophy, especially when induced by pathological stimuli, has been unclear. In this study, NOD2-knockout $(\mathrm{KO})$ mice were used to investigate the role of NOD2 in the hypertrophic response, as well as to

${ }^{1}$ Department of Cardiology, Renmin Hospital of Wuhan University, Wuhan, People's Republic of China and ${ }^{2}$ Cardiovascular Research Institute of Wuhan University, Wuhan, People's Republic of China

Correspondence: Professor Q-z Tang, Department of Cardiology, Renmin Hospital of Wuhan University, Cardiovascular Research Institute of Wuhan University, Jiefang Rd 238, Wuhan 430060, People's Republic of China. E-mail qztang@whu.edu.cn

Received 23 April 2013; revised 18 July 2013; accepted 18 July 2013 
identify the molecular mechanisms that may be responsible for its putative effects.

\section{MATERIALS AND METHODS}

\section{Animal and Animal Models}

All animal procedures were performed in accordance with the Guide for the Care and Use of Laboratory Animals published by the US National Institutes of Health (NIH Publication No. 85-23, revised 1996) and approved by the Animal Care and Use Committee of Renmin Hospital of Wuhan University. Adult male C57/BL6 mice and NOD2-knockout mice (C57BL/6 background) (8-10 weeks old) were used in the current study. NOD2-knockout mice were ordered from the RIKEN BioResource Center, Tsukuba (RBRC), provided by Dr Marco Giovannini. The diet was based on commonly used diets in rodent intervention studies. Aortic banding (AB) was performed as described previously. ${ }^{18}$ Surgery and subsequent analyses were performed in a blinded manner for all groups. Mice were randomly assigned to four groups as WT + sham, $\mathrm{WT}+\mathrm{AB}, \mathrm{KO}+$ sham, and $\mathrm{KO}+\mathrm{AB}$. After the mice were killed, the hearts were dissected and weighed to compare heart weight/body weight (HW/BW, mg/g) and heart weight/ tibia length (HW/TL, $\mathrm{mg} / \mathrm{mm}$ ) ratios in WT and $\mathrm{KO}$ mice.

\section{Echocardiography and Hemodynamics}

Echocardiography was performed using Mylab30CV (ESAOTE S.P.A) with a $10-\mathrm{MHz}$ linear array ultrasound transducer. The LV was assessed in both parasternal long-axis and short-axis views at a frame rate of $50 \mathrm{~Hz}$. End systole and end diastole were defined as the phase in which the smallest and the largest area of LV was obtained, respectively. Left ventricular enddiastolic diameter (LVEDD) and left ventricular end-systolic diameter (LVESD) were measured from the LV M-mode tracing with a sweep speed of $50 \mathrm{~mm} / \mathrm{s}$ at the mid-papillary muscle level.

For hemodynamic measurements, mice were anesthetized with $1.5 \%$ isoflurane, and a microtip catheter transducer (SPR-839, Millar Instruments, Houston, TX, USA) was inserted into the right carotid artery and advanced into the left ventricle. The pressure signals and heart rate were recorded continuously with a Millar pressure-volume system (MPVS400, Millar Instruments, Houston, TX, USA), and the data were processed using PVAN data analysis software.

\section{Histological Analysis}

Hearts were excised, washed with saline solution, and placed in $10 \%$ formalin. Hearts were cut transversely close to the apex to visualize the left and right ventricles. Several sections of the heart (4-5 $\mu \mathrm{m}$ thick) were prepared and stained with H\&E for histopathology or PSR for collagen deposition and visualized using light microscopy. For cardiomyocyte crosssectional area (CSA), sections were stained for membranes with FITC-conjugated WGA (Invitrogen) and for nuclei with DAPI. A single myocyte was measured with a digital quantitative image digital analysis system (Image Pro-Plus, version
6.0). The outline of 100 cardiomyocytes was traced in each group.

\section{Immunofluorescence}

The procedures were described previously. ${ }^{19}$ For immunofluorescence (IF), the sections were blocked with $10 \%$ goat serum, incubated with anti-NOD2 (Santa Cruz Biotechnology, Inc., sc-30199, rabbit) or anti-NF- $\kappa$ Bp65 (Cell Signaling Technology, 4764, rabbit) and anti- $\alpha$-actinin (Millipore, 05-384, mouse) overnight at $4{ }^{\circ} \mathrm{C}$, incubated with Alexa Fluor568 goat anti-rabbit IgG (Invitrogen, A11011) and Alexa Fluor 488 goat anti-mouse IgG (Invitrogen, A11001), and mounted with coverslips using SlowFade Gold antifade reagent with DAPI (Invitrogen, S36939).

\section{Quantitative Real-time PCR and Western Blotting}

Real-time PCR was used to detect those RNA expression levels of hypertrophic and fibrotic markers. Total RNA was extracted from frozen, pulverized mouse cardiac tissue using TRIzol (Roche), and cDNA was synthesized using oligo(dT) primers with the Advantage RT-for-PCR kit (Roche). We performed PCR using LightCycler 480 SYBR Green 1 Master Mix (Roche) and normalized results against glyceraldehyde3-phosphate dehydrogenase (GAPDH) gene expression. For western blotting, cardiac tissues were lysed in RIPA lysis buffer. Fifty micrograms of cell lysate was used for SDSPAGE, and proteins were subsequently transferred to polyvinylidene difluoride membranes (Millipore). Specific protein expression levels were normalized to the GAPDH protein level for total cell lysate and cytosolic proteins on the same polyvinylidene difluoride membrane. The quantification of western blot bands was performed using an Odyssey infrared imaging system (Li-Cor Biosciences). The primary antibodies included antibodies specific for TLR4 (Santa Cruz Biotechnology, Inc., sc30002), p-MEK1/2 (Cell Signaling Technology, 9154), T-MEK1/2 (Cell Signaling Technology, 9122), p-ERK1/2 (Cell Signaling Technology, 4370), T-ERK1/2 (Cell Signaling Technology, 4695), p-P38 (Cell Signaling Technology, 4511), T-P38 (Cell Signaling Technology, 9212), p-JNK (Cell Signaling Technology, 4668), T-JNK (Cell Signaling Technology, 9258), p-90-kDa ribosomal S6 kinase (pp90RSK) (Cell Signaling Technology, 9335), T-p90RSK (Cell Signaling Technology, 9347), p-I $\kappa \mathrm{B} \alpha$ (Cell Signaling Technology, 9246), I $\kappa \mathrm{B} \alpha$ (Cell Signaling Technology, 4814), p-NF$\kappa$ Bp65 (Cell Signaling Technology, 3033), NF- $\kappa$ Bp65 (Cell Signaling Technology, 4764), TNF- $\alpha$ (Cell Signaling Technology, 3707), IL-1 $\beta$ (R\&D, AF-401-NA), IL-10 (R\&D, AF519), MCP-1 (Cell Signaling Technology, 2029), TGF$\beta$ (Abcam, 66043),TGF- $\beta$ ReceptorI(Abcam, ab31013), TGF- $\beta$ Receptor II (Abcam, ab61213), p-Smad2 (Cell Signaling Technology, 8769), Smad2 (Santa Cruz Biotechnology, Inc., sc-6200), p-Smad3 (Cell Signaling Technology, 3101), Smad3 (Santa Cruz Biotechnology, Inc., sc-101154), Smad4 (Santa Cruz Biotechnology, Inc., sc-7966), and GAPDH (Bioworld, MB001). The secondary antibodies anti-rabbit IRdye 800 and 
anti-mouse IRdye 800 (Li-Cor Biosciences) were used at 1:10000 in Odyssey blocking buffer for $1 \mathrm{~h}$. The blots were scanned using an infrared Li-Cor scanner, allowing for the simultaneous detection of two targets (anti-phospho and anti-total protein) in the same experiment.

\section{Statistical Analysis}

Data are expressed as mean \pm s.e.m. Differences among groups were tested by two-way ANOVA followed by a post hoc Tukey test. Comparisons between two groups were performed by unpaired Student's $t$-test. $P<0.05$ was considered to be significantly different.

\section{RESULTS}

\section{Upregulation of NOD2 Expression in Cardiac Tissue Induced by Pressure Overload}

To investigate the response of NOD2 to hypertrophic stresses, we analyzed the mRNA and protein levels of NOD2 in mouse hearts after $A B$ or sham surgery for different durations by real-time PCR and western blot assays. Both mRNA and protein expressions were markedly upregulated after 3 days of $A B$ and were maintained at high levels until 8 weeks after $A B$, but not in the sham group (Figures 1a and b). We explored the localization of NOD2 using immunofluorescence and found that NOD2 could be detected in the cytoplasm of cardiomyocytes in response to pressure overload (Figure 1c). These results indicate that NOD2 expression and activity are enhanced after $\mathrm{AB}$ surgery. NOD2 might have a critical role in the regulation of cardiac remodeling and the transition of cardiac hypertrophy to heart failure.

\section{NOD2 Ablation Exacerbates Cardiac Hypertrophy and Dysfunction in Response to Pressure Overload}

We tested the effect of NOD2 deficiency on cardiac hypertrophy and cardiac function under conditions of pressure overload. We performed $\mathrm{AB}$ surgery or a sham operation in NOD2-KO mice and wild-type (WT) littermates. After 4 weeks, echocardiographic analysis was performed to assess the chamber diameter, wall thickness, and function of the left ventricle. There were no significant changes in the sham group. However, pressure overload induced significantly increased LV mass and poor cardiac function in $\mathrm{KO}$ mice compared with WT mice, as measured by interventricular septal thickness at end diastole (IVSD), left ventricular posterior wall thickness at end diastole (LVPWD), left ventricular end-diastole diameter (LVEDD), left ventricular end-systole diameter (LVESD), ejection fraction (EF), and fractional shortening (FS) (Table 1, Figure 2a). Pressurevolume (PV) loop analysis further confirmed the deteriorated hemodynamic dysfunction of the $\mathrm{LV}$ in response to $\mathrm{AB}$ in $\mathrm{KO}$ mice (Table 1). NOD2-KO mice also showed augmented cardiac hypertrophy 4 weeks after $A B$ evidenced by striking increases in heart weight/body weight ratios, heart weight/tibia length ratios, and cardiomyocyte cross-sectional area (Figure $2 \mathrm{~b}$ ), which were consistent with the morphology of the gross hearts, hematoxylin-eosin (H\&E) staining, and wheat germ agglutinin (WGA) staining (Figure 2c). In addition, the induction of hypertrophic makers, including atrial natriuretic peptide (ANP), B-type natriuretic peptide (BNP), and $\beta$-myosin heavy chain $(\beta$-MHC), was strikingly enhanced in KO mice after pressure overload, accompanied by the downregulation of $\alpha$-myosin heavy chain $(\alpha-\mathrm{MHC})$ and sarcoendoplasmic reticulum Ca2 +-ATPase (SERCA2a) (Figure 2d). These data suggest that NOD2 ablation exacerbated cardiac hypertrophy and deteriorated the impaired cardiac function after pressure overload.

\section{Effects of NOD2 on TLR4 Signaling}

A previous study found that NOD2, in the absence of ligand stimulation, has a negative role in TLR4-mediated signaling pathways. $^{20}$ We found that the mRNA and protein expressions of TLR4 were increased in mice 4 weeks after $\mathrm{AB}$, especially in the NOD2-KO group (Figures $3 \mathrm{a}$ and $\mathrm{b}$ ). MAPK and NF- $\kappa$ B signaling was downstream of NOD2 and TLR4. ${ }^{14}$ To explore the molecular mechanism by which NOD2 affects the hypertrophic response, the activation of MAPK in KO and WT hearts induced by pressure overload was assessed using western blot. AB mice displayed increased phosphorylation of mitogen-activated protein kinase $1 / 2$ (MEK), extracellular signal-regulated kinase (ERK) 1/2, c-Jun $\mathrm{N}$-terminal kinase (JNK), p38, and p90RSK. NOD2 deficiency enhanced the phosphorylation of MEK-ERK1/2, p38, and p90RSK. However, we did not find a difference in phosphorylated JNK levels between $\mathrm{KO}$ and WT mice (Figure 3c). Previous studies suggest that the activation of $\mathrm{NF}-\kappa \mathrm{B}$ signaling has an important role in the pathogenesis of cardiac remodeling and heart failure; ${ }^{21,22}$ therefore, we examined NF- $\kappa \mathrm{B}$ signaling in the mice. Nuclear translocation of NF- $\kappa \mathrm{Bp} 65$ protein was dramatically enhanced in the cardiomyocytes of hypertrophic hearts after $\mathrm{AB}$, especially in $\mathrm{KO}$ mice (Figure $3 \mathrm{~d}$ ). The phosphorylation of $\mathrm{I} \kappa \mathrm{B} \alpha$ and NF- $\kappa$ Bp 65 was significantly activated, whereas the total level of $\mathrm{I} \kappa \mathrm{B} \alpha$ was lower in $\mathrm{KO}$ mice after $\mathrm{AB}$ compared with WT mice (Figure 3e). We also examined the expression of NF- $\kappa \mathrm{B}$ target genes, including interleukin (IL)-1 $\beta$, IL-10, tumor necrosis factor (TNF)- $\alpha$, and monocyte chemoattractant protein 1 (MCP-1), in the cardiac tissue of KO mice and their WT littermates 4 weeks after $A B$. Our results showed that both mRNA and protein levels of IL-1 $\beta$, IL-10, TNF- $\alpha$, and MCP-1 increased in KO mice compared with WT mice after $\mathrm{AB}$ (Figure $3 \mathrm{f}$ and $\mathrm{g}$ ). These results indicate that NOD2 inhibited cardiac hypertrophy by blocking NF- $\kappa \mathrm{B}$ signaling in response to chronic pressure overload.

\section{NOD2 Inhibits Cardiac Fibrosis after Pressure Overload}

Cardiac fibrosis is a hallmark of most cardiac pathologies. Left ventricular interstitial fibrosis was evaluated using picrosirius red (PSR) on paraffin-embedded sections. Perivascular and interstitial fibrosis was detected in both $\mathrm{KO}$ and WT mice after pressure overload, but the extent of cardiac 
a

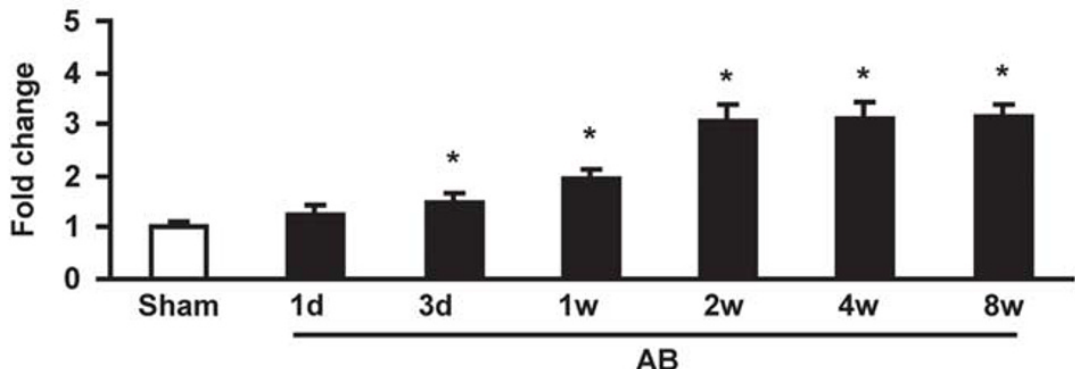

b
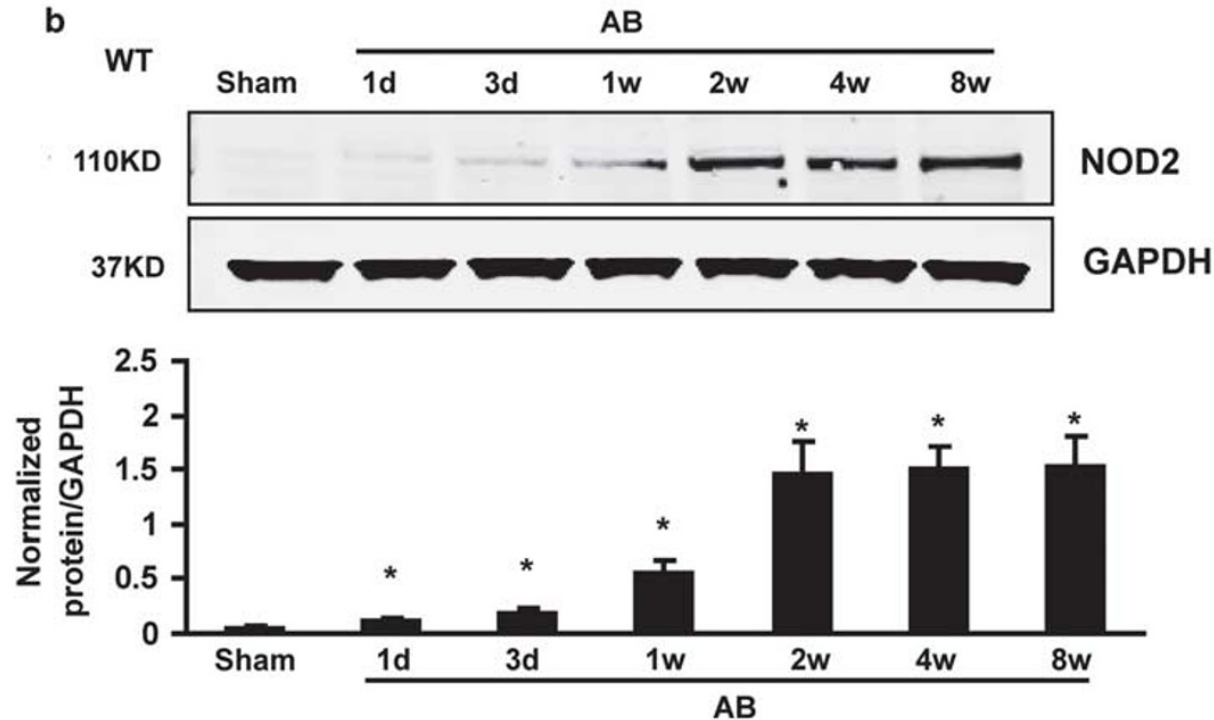

C

NOD2
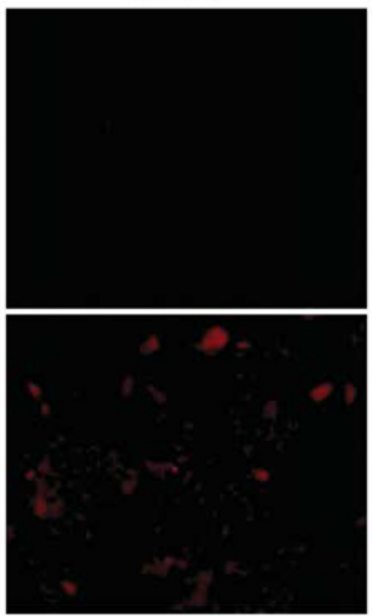

$\alpha$-actin
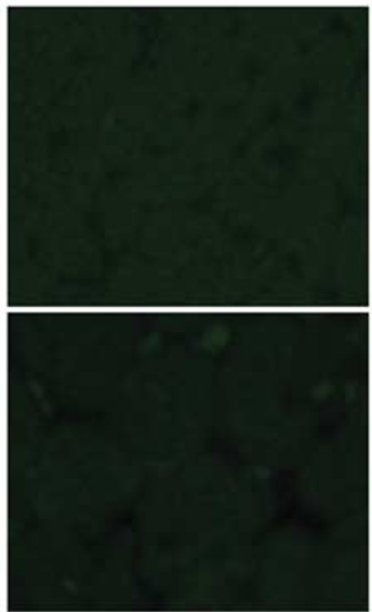

DAPI
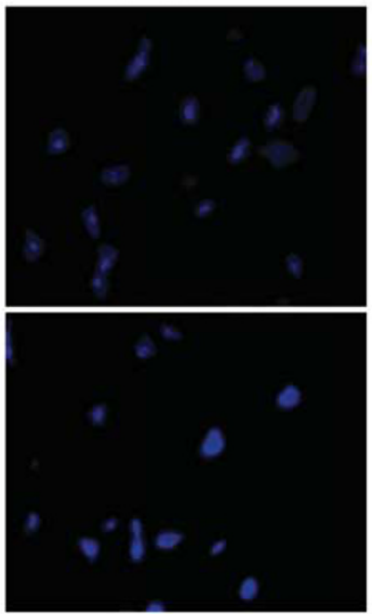
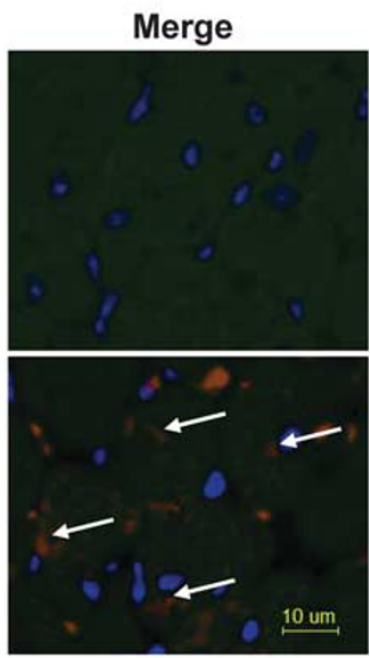

Figure 1 NOD2 expression in the hypertrophic heart. (a) The expression of NOD2 determined by real-time PCR analysis from WT mice after aortic banding at the time points indicated $(n=6) .{ }^{*} P<0.05$ vs sham. (b) Western blot analysis of cardiac NOD2 protein from WT mice after aortic banding at the time points indicated $(n=6)$. Top, representative blots; bottom, quantitative results. ${ }^{*} P<0.05$ vs sham. (c) Immunofluorescence of cardiac NOD2 protein from WT mice after aortic banding at the time points indicated.

fibrosis was markedly enhanced in $\mathrm{KO}$ mice induced by pressure overload (Figures $4 \mathrm{a}$ and $\mathrm{b}$ ). We found that the mRNA expression of fibrotic makers including TGF- $\beta 1$, TGF- $\beta 2$, collagen I $\alpha$, collagen III, and connective tissue growth factor (CTGF) was remarkably upregulated in KO mice compared with WT mice in response to $\mathrm{AB}$ (Figure 4c). To further illustrate the cellular mechanisms underlying the antifibrotic effects of NOD2, we assessed the regulatory role 
Table 1 Echocardiographic and hemodynamic parameters in NOD2 KO mice 4 weeks after surgery

$\begin{array}{lcccc} & \text { Sham } & \text { Sham } & \text { AB } & \text { AB } \\ \text { Parameter } & \text { WT } & \text { KO } & \text { WT } & \text { KO } \\ & & & & \\ & n=9 & n=8 & n=10 & n=10 \\ \text { IVSD }(\mathrm{mm}) & 0.68 \pm 0.02 & 0.68 \pm 0.01 & 0.76 \pm 0.01^{*} & 0.81 \pm 0.01^{\#} \\ \text { LVPWD }(\mathrm{mm}) & 0.70 \pm 0.02 & 0.68 \pm 0.01 & 0.75 \pm 0.01^{*} & 0.78 \pm 0.01^{\#} \\ \text { LVEDD }(\mathrm{mm}) & 3.63 \pm 0.05 & 3.60 \pm 0.03 & 4.12 \pm 0.06^{*} & 4.51 \pm 0.05^{\#} \\ \text { LVESD }(\mathrm{mm}) & 2.11 \pm 0.03 & 2.14 \pm 0.03 & 2.70 \pm 0.05^{*} & 3.15 \pm 0.06^{\#} \\ \text { EF(\%) } & 78.7 \pm 0.71 & 77.3 \pm 0.60 & 69.7 \pm 0.88^{*} & 60.0 \pm 1.20^{\#} \\ \text { FS(\%) } & 41.2 \pm 0.61 & 40.0 \pm 0.55 & 34.1 \pm 0.75^{*} & 30.0 \pm 0.73^{\#} \\ & n=7 & n=6 & n=7 & n=6 \\ \text { ESP }(\mathrm{mm} \mathrm{Hg}) & 112 \pm 3.11 & 115 \pm 3.98 & 154 \pm 2.79^{*} & 152 \pm 5.24^{*} \\ \mathrm{~d} p / \mathrm{d} t_{\max }(\mathrm{mm} \mathrm{Hg} / \mathrm{s}) & 8983 \pm 388 & 8718 \pm 196 & 7980 \pm 390^{*} & 7478 \pm 388^{\#} \\ \mathrm{~d} p / \mathrm{d} t_{\min }(\mathrm{mm} \mathrm{Hg} / \mathrm{s}) & -8657 \pm 200-8633 \pm 455 & -7329 \pm 345^{*} & -7087 \pm 519^{\#} \\ & & & & \end{array}$

Abbreviations: $\mathrm{d} p / \mathrm{d} t_{\max }$, maximal rate of pressure development; $\mathrm{dp} / \mathrm{d} t_{\min }$ minimal rate of pressure decay; EF, ejection fraction; ESP, end-systolic pressure; FS, fractional shortening; IVSD, interventricular septal thickness at end diastole; LVEDD, left ventricular end-diastolic diameter; LVESD, left ventricular end-systolic diameter; LVPWD, left ventricular posterior wall thickness at end diastole.

${ }^{*} P<0.05$ for difference from the corresponding sham group. ${ }^{\#} P<0.05$ vs the $W T+A B$ group.

of NOD2 in TGF- $\beta /$ Smad cascade activation. The protein levels of TGF- $\beta$, TGF- $\beta$ RI, TGF- $\beta$ RII, p-Smad2, p-Smad3, and Smad4 were increased in $\mathrm{KO}$ mice after $\mathrm{AB}$ surgery (Figures $4 \mathrm{~d}$ and e). Collectively, these results indicate that NOD2 deficiency exaggerated fibrosis in response to the pathological pressure overload by promoting TGF- $\beta / \mathrm{Smad}$ signaling.

\section{DISCUSSION}

In this study, we used NOD2-KO mice to examine the role of NOD2 in the hypertrophic response. The results demonstrate that NOD2 disruption aggravated cardiac hypertrophy and fibrosis under conditions of pressure overload. The effects of NOD2 on cardiac hypertrophy and fibrosis are likely mediated by the regulation of TLR 4 and TGF- $\beta /$ Smad signaling. These results suggest that NOD2 may have a protective role in pathological hypertrophy in the heart and may be an effective therapeutic candidate for cardiac hypertrophy and heart failure.

NOD2 is an intracellular receptor for the bacterial cell wall component muramyl dipeptide, ${ }^{12,13}$ and contributes to innate antimycobacterial immunity. ${ }^{23}$ NOD2 signaling has an important role in inflammation. ${ }^{11,24}$ NOD2 may represent a new therapeutic target in liver diseases. ${ }^{25}$ However, the biochemical mechanism by which NOD2 mediates its antihypertrophic effects remains elusive. The development of cardiac hypertrophy is complex. Therefore, it is necessary to demonstrate the mechanism of cardiac hypertrophy and find the key targets for the treatment of pathological cardiac hypertrophy.

NOD2 was upregulated during liver injury in mice and humans, and mice-lacking NOD2 were protected from ConA-induced hepatitis. ${ }^{25}$ The NOD2 upregulation in atheroma lesions indicates an involvement of this protein in the pathology of $\mathrm{CHD}^{26}$ Our data showed that cardiac NOD2 increased after $A B$ surgery in the cardiomyocyte cytoplasm, which suggests that the upregulated NOD2 is involved in $\mathrm{AB}$-induced hypertrophy (Figure 1).

Cardiac hypertrophy is largely characterized by increased size and/or thickness of the left ventricle. Particularly, the cardiomyocytes expand in size and increase their protein synthesis. ${ }^{27}$ Echocardiography and pressure-volume loops provide a framework for understanding cardiac mechanics in experimental animals and humans. In this study, a significantly larger IVSD, LVPWD, LVEDD, and LVESD of $\mathrm{KO}$ mice on echocardiographs 4 weeks after $\mathrm{AB}$ operation supported the increased LV mass and LV dilation (Table 1, Figure 2a). The decreased EF and FS of KO mice induced by pressure overload suggest poor cardiac function in $\mathrm{KO}$ mice compared with WT mice (Table 1, Figure 2a). $\pm \mathrm{d} p / \mathrm{d} t_{\max }$ and $\pm \mathrm{d} p / \mathrm{d} t_{\min }$ are much more accurate indices in discussing cardiac systolic and diastolic function. ${ }^{28,29}$ In this study, LV systolic pressures were similar between $\mathrm{KO}$ and WT mice 4 weeks after $A B$, being statistically higher than shams. Pressure overload significantly decreased $\pm \mathrm{d} p / \mathrm{d} t_{\max }$ and $\pm \mathrm{d} p / \mathrm{d} t_{\min }$ in KO mice compared with WT mice (Table 1). HW/BW, HW/TL, and CSA are usually used to evaluate the degree of hypertrophy. In this study, the $\mathrm{KO}$ mice exhibited a significantly higher HW/BW, HW/TL, and CSA, which should be attributed to the increased afterload and the impaired cardiac function caused by pressure overload (Figures $2 \mathrm{~b}$ and c). In response to hypertrophic stimuli, a fundamental reprogramming occurs within the adult cardiomyocyte that results in an altered profile of gene expression. Genes such as $\beta$-MHC, BNP, and atrial natriuretic factor (ANF) become highly expressed within ventricular myocytes..$^{30,31}$ In the rat model of pressure-overload hypertrophy induced by ascending aortic banding, the decrease in SERCA2a mRNA content parallels decrease in SERCA2a protein concentration and Ca2 + -ATPase activity. ${ }^{32,33}$ Our results were consistent with previous studies. The mRNA levels of ANP, BNP, and $\beta$-MHC were increased in $\mathrm{KO}$ mice after pressure overload, accompanied by the downregulation of $\alpha$-MHC and SERCA2a (Figure 2d). These results suggest that NOD2 deletion exacerbates cardiac hypertrophy and deteriorates impaired cardiac function after pressure overload.

A previous study represents a novel mechanism linking NOD2 activation with the inhibition of TLR4 signaling in enterocytes in vivo and in vitro. ${ }^{34}$ Thus, we analyzed the effects of NOD2 on TLR4 in the heart. The results showed that the mRNA and protein expressions of TLR4 were increased in mice 4 weeks after $\mathrm{AB}$, especially in the NOD2 
a

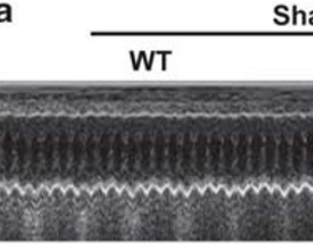

Sham

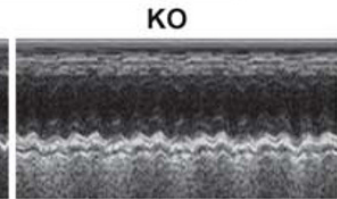

$\mathrm{AB}$

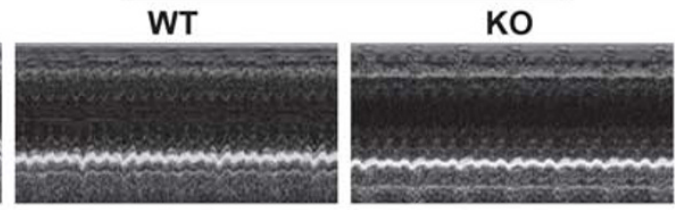

b
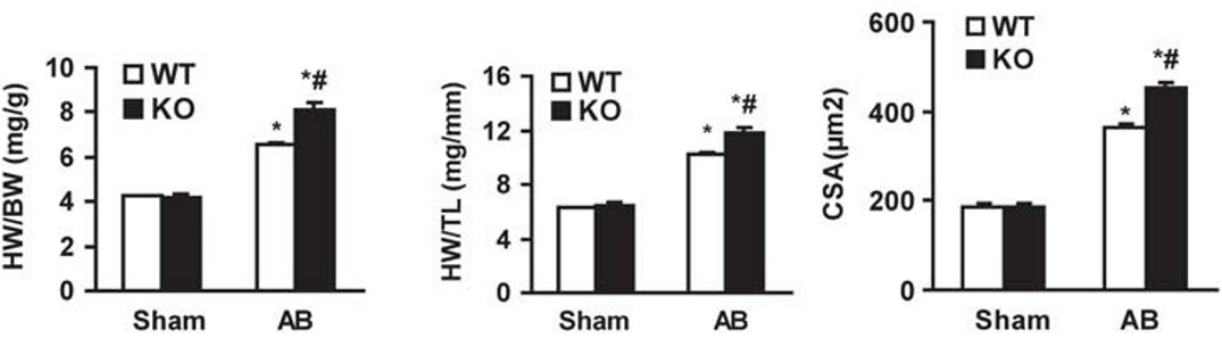

C

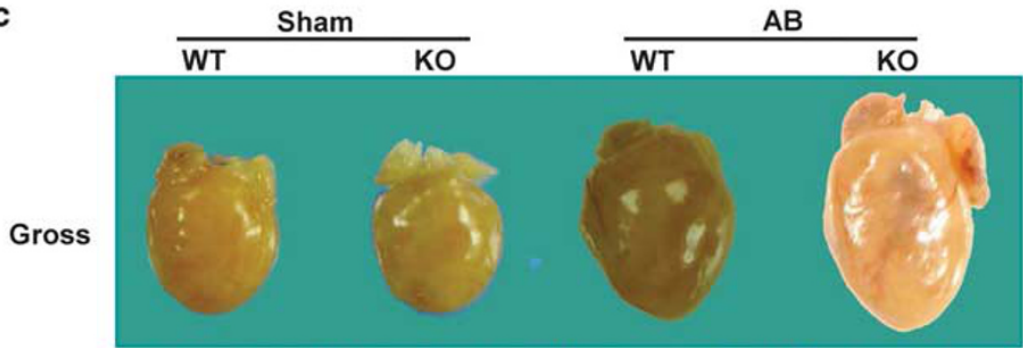

HE
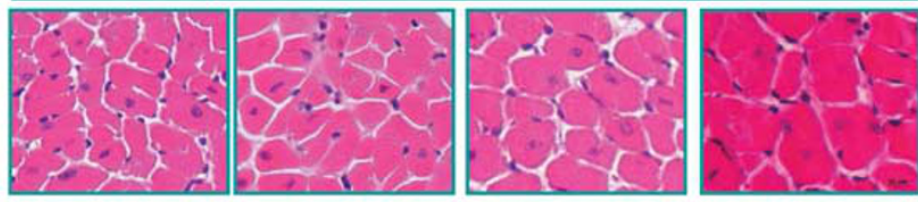

WGA
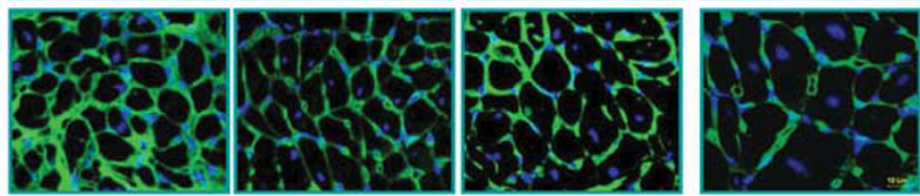

d
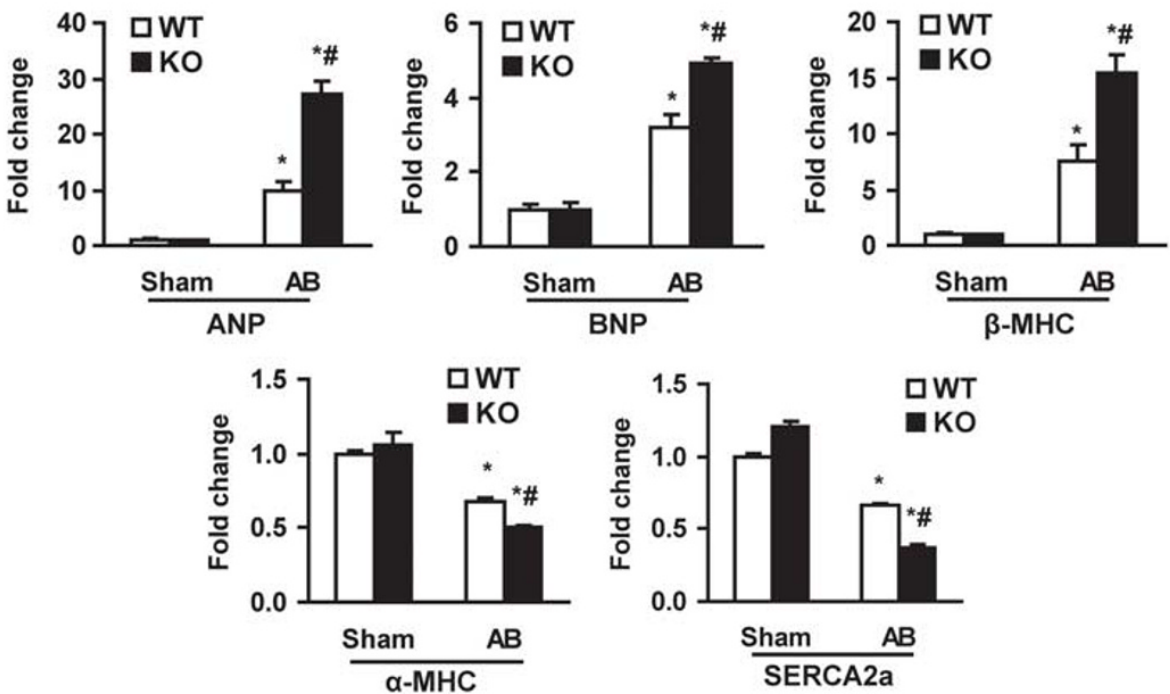

Figure 2 Effects of NOD2 deficiency on cardiac hypertrophy. (a) M-mode echocardiogram from the four groups of mice 4 weeks after AB or sham surgery. (b) Statistical results of the HW/BW, HW/TL $(n=9-16)$, and myocyte cross-sectional areas of the indicated groups. (c) Gross hearts, H\&E staining, and WGA-FITC staining of sham and AB mice at 4 weeks post surgery. (d) Expressions of transcripts for ANP, BNP, $\beta$-MHC, $\alpha$-MHC, and SERCA2a induced by AB were determined by real-time PCR analysis $(n=6) .{ }^{*} P<0.05$ compared with the corresponding sham group. ${ }^{\#} P<0.05$ vs the $\mathrm{WT}+\mathrm{AB}$ group. 
a
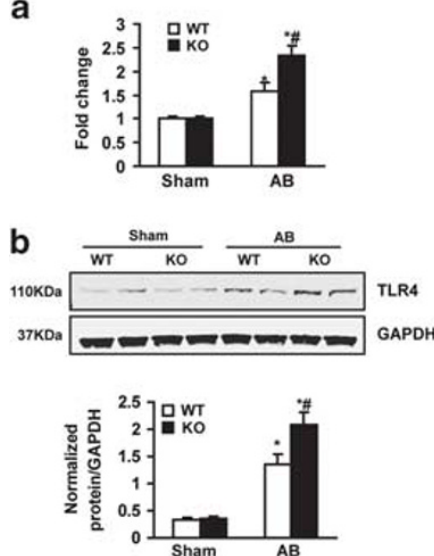

C

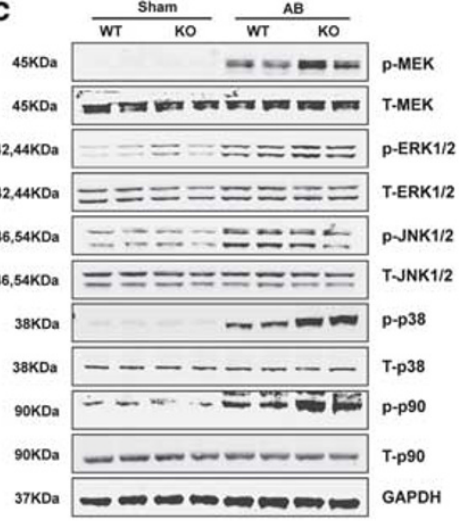

d

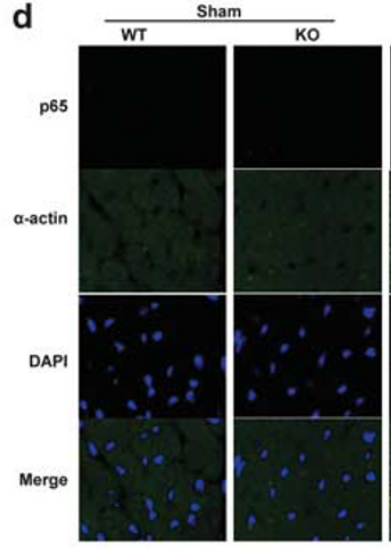

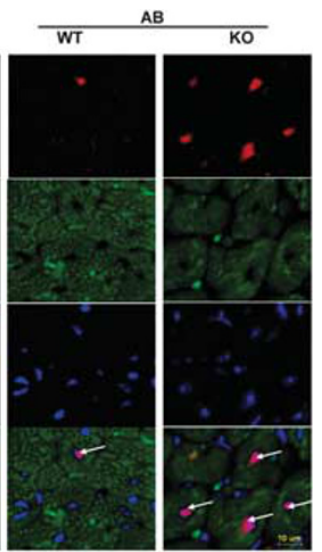

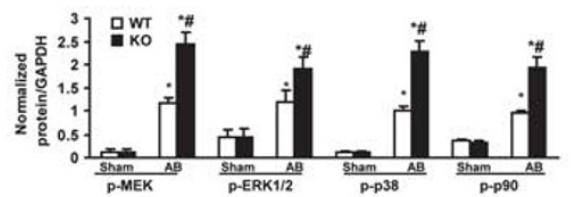

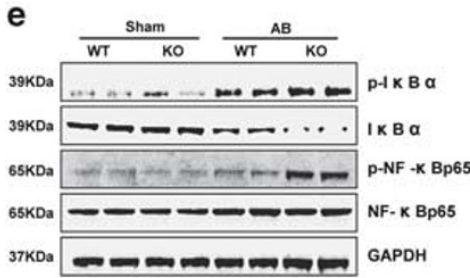

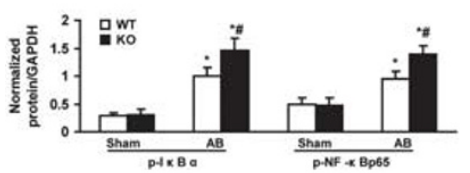

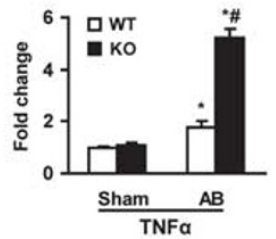
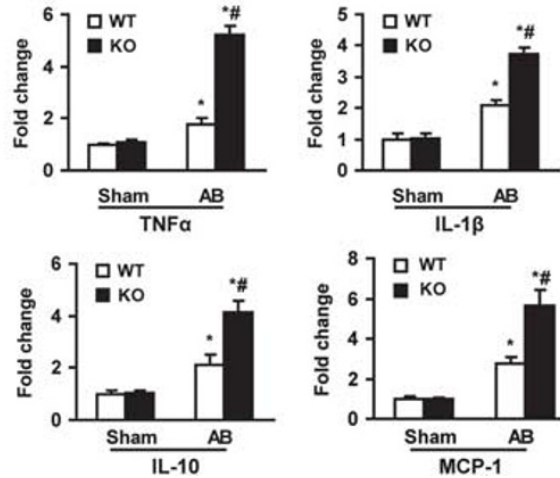

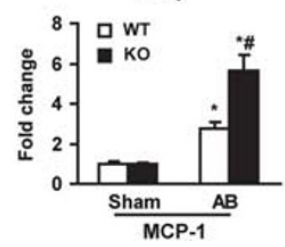

g
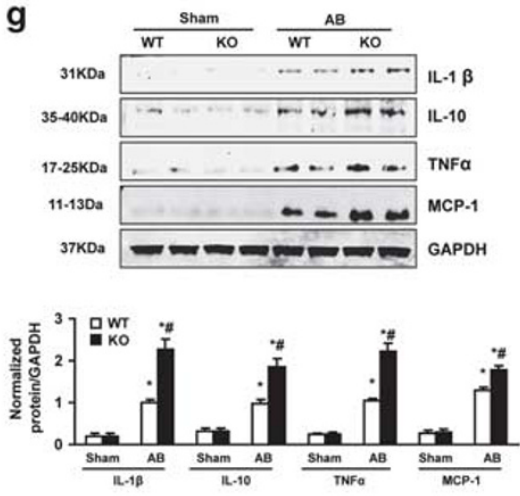

Figure 3 Effects of NOD2 on TLR4 signaling. (a) The mRNA expression levels of TLR4 in hearts of each group 4 weeks after AB or sham surgery ( $n=6$ ). (b) Western blot analysis of cardiac TLR4 protein in each group 4 weeks after AB or sham surgery. Top, representative blots; bottom, quantitative results $(n=6)$. (c) The levels of total and phosphorylated MEK, ERK1/2, JNK1/2, p38, and p90RSK in mice from the indicated groups ( $n=6)$. Top, representative blots; bottom, quantitative results. (d) Representative of immunofluorescence staining of cardiac NF- $\kappa$ Bp65 protein expression in each group 4 weeks after $A B$ or sham surgery. A white arrow indicates nuclear-translocated NF- $\kappa$ Bp65. (e) Western blot analysis of total and phosphorylated $I \kappa \mathrm{B}$ and NF- $\kappa \mathrm{Bp} 65$ in the heart tissues of mice in the indicated groups $(n=6)$. Top, representative blots; bottom, quantitative results. (f) The mRNA expressions of IL-1 $\beta, \mathrm{IL}-10, \mathrm{TNF}-\alpha$, and MCP-1 in the myocardium were obtained from indicated groups using real-time PCR analysis $(n=6)$. $(\mathbf{g})$ Western blot analysis of IL-1 $\beta$, IL-10, TNF- $\alpha$, and MCP-1 in the myocardium of the indicated groups $(n=6)$. Top, representative blots; bottom, quantitative results. ${ }^{*} P<0.05$ compared with the corresponding sham group. ${ }^{\#} P<0.05$ vs the $W T+A B$ group.

ablation group (Figures 3a and b). Increased TLR4 expression has been observed in human heart failure and ischemic hearts, ${ }^{35}$ and reduced cardiac hypertrophy in TLR4-deficient mice following aortic banding. ${ }^{36}$ Our data indicate that loss of NOD2 may impair the resistant ability of heart under stress mainly through TLR4 signaling. The TLR and NOD pathways share many similarities in downstream signaling pathways, such as activation of MAPKs and of NF- $\kappa$ B. ${ }^{14}$ The MAPK family includes extracellular signal receptor-regulated kinase (ERK), c-jun NH2-terminal Kinase (JNK), and p38 MAPK, which have been reported to be important for the induction of hypertrophic responses. ${ }^{37}$ Previous studies suggest that NOD2 activation results in phosphorylation of p38, JNK, and ERK in immature human dendritic cells. ${ }^{38}$ NOD2 - / - mice demonstrate decreased ERK, p38, and I $\kappa \mathrm{B}$ phosphorylation on MDP stimulation, confirming the role of NOD2 role in the activation of MAPK and NF- $\kappa \mathrm{B}$ pathways. $^{39}$ However, here we showed that NOD2 negatively regulated ERK and p38 activation in the hypertrophic heart, which seems to be inconsistent with previous data (Figure 3c). A potential reason for the discrepancy is that the effect of NOD2 on the ERK pathway depends on TLR4 activation. The p90 ribosomal S6 kinase (RSK) constitutes a family of serine/threonine kinases activated downstream of the MAPK pathway. Increasing evidence showed that ERK1/2 activation leads to the activation 
a
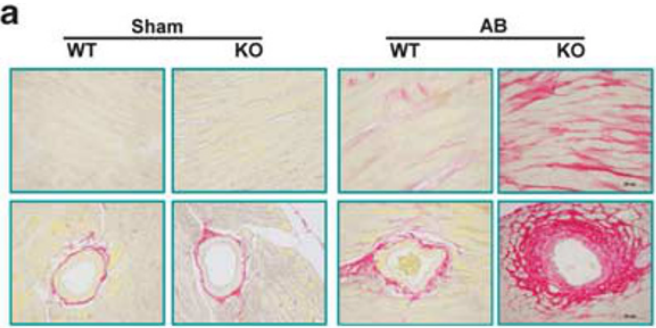

b
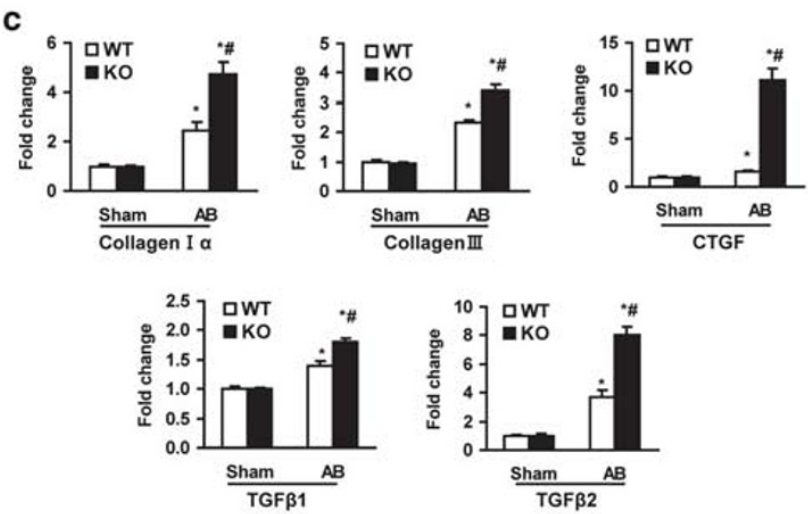

d

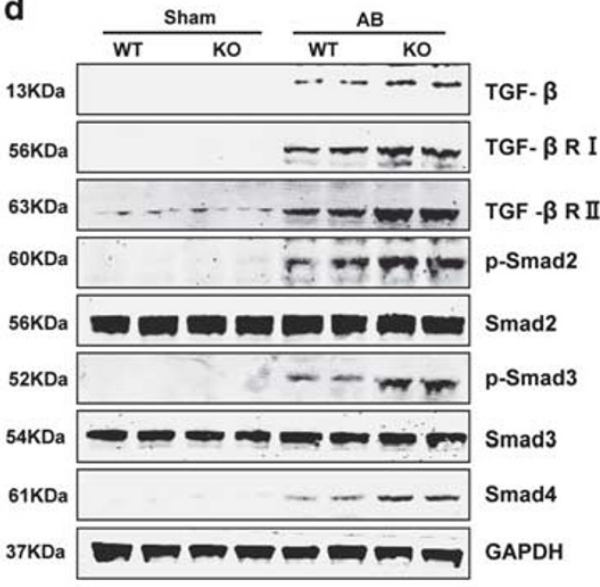

Figure 4 NOD2 attenuated the fibrosis induced by pressure overload. (a) PSR staining of histological sections of the LV was performed in each group 4 weeks after $A B$ or sham surgery. (b) Fibrotic areas of the histological sections were quantified using an image-analyzing system. (c) The mRNA expressions of TGF- $\beta 1$, TGF- $\beta 2$, collagen I $\alpha$, collagen III, and CTGF in the myocardium were obtained from indicated groups using real-time PCR analysis $(n=6)$. (d, e) Western blot analysis of the levels of TGF- $\beta$, TGF- $\beta$ RI, TGF- $\beta$ RII, p-Smad2, Smad2, p-Smad3, Smad3, and Smad4 in mice from indicated groups $(n=6)$. (d) Representative blots. (e) Quantitative results. ${ }^{*} P<0.05$ compared with the corresponding sham group. ${ }^{\#} P<0.05$ vs the WT + AB group.

of the transcription factor, p90RSK. ${ }^{40}$ We showed that NOD2 disruption enhanced p-p90RSK activation induced by pressure overload, and that such effect is dependent on ERK1/2 signaling (Figure 3c).

Mounting evidence has strongly suggested that NF- $\kappa \mathrm{B}$ signaling has a key role in the development of cardiac hypertrophy and heart failure. ${ }^{7,8}$ A previous study found that many signaling cascades induced by LPS (NF- $\kappa \mathrm{B}$, IKK, MAPKs, and STAT1) increased in NOD2-knockdown cells. ${ }^{20}$ These unexpected results imply that NOD2 might have a role in the negative regulation of LPS-induced inflammatory responses. ${ }^{11}$ In line with the growing evidence, our results show that NOD2 significantly abrogated NF- $\kappa$ B activation by disrupting transcriptional activity. This was done by blocking the phosphorylation and degradation of $\mathrm{I} \kappa \mathrm{B} \alpha$ as well as by inhibiting the expression of NF- $\kappa \mathrm{B}$ target genes (IL- $1 \beta$, IL-10, TNF- $\alpha$, and MCP-1) in hypertrophic hearts (Figures $3 \mathrm{~d}-\mathrm{g}$ ).

Fibrosis is another classical feature of pathological hypertrophy, which is characterized by the expansion of the extracellular matrix by the accumulation of collagen. Thus, it is important to understand the mechanisms that stimulate collagen deposition in the heart and define approaches to limit these processes. In this study, we analyzed the anti-fibrotic properties of NOD2 in the heart. Perivascular and interstitial fibrosis was detected in both $\mathrm{KO}$ and WT mice. The extent of cardiac fibrosis was markedly enhanced in $\mathrm{KO}$ mice 4 weeks after $\mathrm{AB}$ (Figures $4 \mathrm{a}$ and $\mathrm{b}$ ). The mRNA expressions of TGF- $\beta 1$, TGF- $\beta 2$, collagen I $\alpha$, collagen III, and CTGF, which are responsible for cardiac fibrosis, were remarkably upregulated in the $\mathrm{KO}$ mice compared with the WT mice in response to $\mathrm{AB}$ (Figure 4c). In an attempt to elucidate the mechanisms underlying the inhibitory effect of NOD2 on fibrosis, we examined mediators of fibrosis and TGF- $\beta /$ Smad signaling, which has an important role in the progression of fibrosis. ${ }^{8}$ TGF- $\beta$ signaling is transduced through Smad and non-Smad pathways. TGF- $\beta$ ligand binds to TGF- $\beta$ R 2 and TGF- $\beta$ R 1 . TGF- $\beta$ R 2 phosphorylates (P) TGF- $\beta$ R1, which subsequently phosphorylates and activates $S m a d 2$ and Smad3. Activated Smad2 and Smad3 form a Smad complex with Smad4, and it translocates into the nucleus. ${ }^{41}$ Our data showed that NOD2 disruption enhanced TGF- $\beta /$ Smad signaling activation induced by AB (Figure $4 d$ ). Collectively, these data indicate that NOD2 attenuates fibrosis by blocking TGF- $\beta /$ Smad signaling.

In conclusion, our present work demonstrates that NOD2 inhibits cardiac hypertrophy and fibrosis in response to hypertrophic stimuli in mice by blocking TLR 4 and TGF- $\beta$ / Smad signaling, and by regulation of pro-fibrotic cytokines and collagen content. This study enhances our understanding of the inhibitory effect of NOD2 on cardiac hypertrophy and the related molecular mechanisms. It also serves to elucidate the dominant signaling pathways leading to cardiac hypertrophy and fibrosis in response to hypertrophic stimuli. 


\section{ACKNOWLEDGEMENTS}

We thank Dr Marco Giovannini at Inserm U674 and Institut Universitaire d'hématologie of Université Paris 7-Denis Diderot for providing the NOD2$\mathrm{KO}$ mice. This work was supported by the National Nature Science Foundation of China (30901628,30972954,81000036 and 81000095) and the Fundamental Research Funds for the Central Universities of China $(5107002$ and 2012302020212).

\section{DISCLOSURE/CONFLICT OF INTEREST}

The authors declare no conflict of interest.

1. Lorell BH, Carabello BA. Left ventricular hypertrophy: pathogenesis, detection, and prognosis. Circulation 2000;102:470-479.

2. Ho KK, Pinsky JL, Kannel WB, et al. The epidemiology of heart failure: the Framingham Study. J Am Coll Cardiol 1993;22(4 Suppl A):6A-13A.

3. Lloyd-Jones DM, Larson MG, Leip EP, et al. Lifetime risk for developing congestive heart failure: the Framingham Heart Study. Circulation 2002;106:3068-3072.

4. Maillet M, Lynch JM, Sanna B, et al. Cdc42 is an antihypertrophic molecular switch in the mouse heart. J Clin Invest 2009;119:3079-3088.

5. Rose BA, Force T, Wang Y. Mitogen-activated protein kinase signaling in the heart: angels versus demons in a heart-breaking tale. Physio Rev 2010;90:1507-1546.

6. Aoyagi $T$, Matsui T. Phosphoinositide-3 kinase signaling in cardiac hypertrophy and heart failure. Curr Pharm Des 2011;17:1818-1824.

7. Balakumar P, Jagadeesh G. Multifarious molecular signaling cascades of cardiac hypertrophy: can the muddy waters be cleared? Pharmacol Res 2010;62:365-383.

8. Ai W, Zhang Y, Tang QZ, et al. Silibinin attenuates cardiac hypertrophy and fibrosis through blocking EGFR-dependent signaling. J Cell Biochem 2010;110:1111-1122.

9. Inohara N, Nunez G. NODs: intracellular proteins involved in inflammation and apoptosis. Nat Rev Immunol 2003;3:371-382.

10. Chamaillard M, Girardin SE, Viala J, et al. Nods, Nalps and Naip: intracellular regulators of bacterial-induced inflammation. Cell Microbiol 2003;5:581-592.

11. Watanabe T, Kitani A, Murray PJ, et al. NOD2 is a negative regulator of Toll-like receptor 2-mediated T helper type 1 responses. Nat Immunol 2004;5:800-808

12. Inohara N, Ogura $\mathrm{Y}$, Fontalba $\mathrm{A}$, et al. Host recognition of bacterial muramyl dipeptide mediated through NOD2. Implications for Crohn's disease. J Biol Chem 2003;278:5509-5512.

13. Girardin SE, Boneca IG, Viala J, et al. Nod2 is a general sensor of peptidoglycan through muramyl dipeptide (MDP) detection. J Biol Chem 2003;278:8869-8872.

14. Abraham C, Cho JH. Functional consequences of NOD2 (CARD15) mutations. Inflamm Bowel Dis 2006;12:641-650.

15. Hugot JP, Chamaillard M, Zouali $\mathrm{H}$, et al. Association of NOD2 leucinerich repeat variants with susceptibility to Crohn's disease. Nature 2001;411:599-603.

16. Miceli-Richard C, Lesage S, Rybojad M, et al. CARD15 mutations in Blau syndrome. Nat Genet 2001;29:19-20.

17. Shaw MH, Reimer T, Sanchez-Valdepenas C, et al. T cell-intrinsic role of Nod2 in promoting type 1 immunity to Toxoplasma gondii. Nat Immunol 2009;10:1267-1274.

18. Yan L, Wei X, Tang QZ, et al. Cardiac-specific mindin overexpression attenuates cardiac hypertrophy via blocking AKT/GSK3beta and TGF-beta1-Smad signalling. Cardiovasc Res 2011;92:85-94.

19. Zhou H, Bian ZY, Zong J, et al. Stem cell antigen 1 protects against cardiac hypertrophy and fibrosis after pressure overload. Hypertension 2012;60:802-809.
20. Tsai WH, Huang DY, Yu YH, et al. Dual roles of NOD2 in TLR4-mediated signal transduction and -induced inflammatory gene expression in macrophages. Cell Microbiol 2011;13:717-730.

21. Yan L, Huang H, Tang QZ, et al. Breviscapine protects against cardiac hypertrophy through blocking PKC-alpha-dependent signaling. J Cell Biochem 2010;109:1158-1171.

22. Shen DF, Tang QZ, Yan L, et al. Tetrandrine blocks cardiac hypertrophy by disrupting reactive oxygen species-dependent ERK1/2 signalling. $\mathrm{Br}$ J Pharmacol 2010;159:970-981.

23. Divangahi M, Mostowy S, Coulombe F, et al. NOD2-deficient mice have impaired resistance to Mycobacterium tuberculosis infection through defective innate and adaptive immunity. J Immunol 2008;181: 7157-7165.

24. Ferwerda G, Girardin SE, Kullberg BJ, et al. NOD2 and toll-like receptors are nonredundant recognition systems of Mycobacterium tuberculosis. PLoS Pathog 2005;1:279-285.

25. Body-Malapel M, Dharancy S, Berrebi D, et al. NOD2: a potential target for regulating liver injury. Lab Invest 2008:88:318-327.

26. El Mokhtari NE, Ott SJ, Nebel A, et al. Role of NOD2/CARD15 in coronary heart disease. BMC Genet 2007;8:76.

27. Selvetella G, Hirsch E, Notte A, et al. Adaptive and maladaptive hypertrophic pathways: points of convergence and divergence. Cardiovasc Res 2004;63:373-380.

28. Chen TM, Li J, Liu L, et al. Effects of heme oxygenase-1 upregulation on blood pressure and cardiac function in an animal model of hypertensive myocardial infarction. Int J Mol Sci 2013;14:2684-2706.

29. Zong J, Deng W, Zhou H, et al. 3,3'-Diindolylmethane protects against cardiac hypertrophy via 5 '-adenosine monophosphate-activated protein kinase-alpha2. PLoS ONE 2013;8:e53427.

30. van Berlo JH, Elrod JW, van den Hoogenhof MM, et al. The transcription factor GATA-6 regulates pathological cardiac hypertrophy. Circ Res 2010;107:1032-1040.

31. Heineke J, Molkentin JD. Regulation of cardiac hypertrophy by intracellular signalling pathways. Nat Rev Mol Cell Biol 2006;7:589-600.

32. Feldman $\mathrm{AM}$, Weinberg $\mathrm{EO}$, Ray $\mathrm{PE}$, et al. Selective changes in cardiac gene expression during compensated hypertrophy and the transition to cardiac decompensation in rats with chronic aortic banding Circ Res 1993;73:184-192.

33. Miyamoto Ml, del Monte $\mathrm{F}$, Schmidt $\mathrm{U}$, et al. Adenoviral gene transfer of SERCA2a improves left-ventricular function in aortic-banded rats in transition to heart failure. Proc Natl Acad Sci USA 2000;97:793-798.

34. Richardson WM, Sodhi CP, Russo A, et al. Nucleotide-binding oligomerization domain-2 inhibits toll-like receptor-4 signaling in the intestinal epithelium. Gastroenterology 2010;139:904-917, 17 e1-6.

35. Frantz S, Kobzik L, Kim YD, et al. Toll4 (TLR4) expression in cardiac myocytes in normal and failing myocardium. J Clin Invest 1999; 104:271-280

36. Ha T, Li Y, Hua F, et al. Reduced cardiac hypertrophy in toll-like receptor 4-deficient mice following pressure overload. Cardiovasc Res 2005;68:224-234.

37. Rohini A, Agrawal N, Koyani CN, et al. Molecular targets and regulators of cardiac hypertrophy. Pharmacol Res 2010;61:269-280.

38. Vidal V, Dewulf J, Bahr GM. Enhanced maturation and functional capacity of monocyte-derived immature dendritic cells by the synthetic immunomodulator Murabutide. Immunology 2001;103: 479-487.

39. Kobayashi KS, Chamaillard $M$, Ogura $Y$, et al. Nod2-dependent regulation of innate and adaptive immunity in the intestinal tract. Science 2005:307:731-734.

40. Romeo Y, Zhang X, Roux PP. Regulation and function of the RSK family of protein kinases. Biochem J 2012;441:553-569.

41. Ikushima $\mathrm{H}$, Miyazono K. TGFbeta signalling: a complex web in cancer progression. Nat Rev Cancer 2010;10:415-424. 